\title{
Title: Asleep at the Wheel: Forward Genetic ENU Mutagenesis Screen for Mouse Models of Chronic Fatigue Identifies a Mutation in SIc2a4 (GLUT4)
}

\author{
Short Title: Chronic Fatigue Screen Identifies Scl2a4 Mutation
}

Marleen H. M. de Groot ${ }^{a, b}$, Carlos M. Castorena ${ }^{c}$, Vivek Kumar ${ }^{a, 1}$, Jennifer A. Mohawk $^{\mathrm{a}, \mathrm{b}}$, Newaz I. Ahmed ${ }^{\mathrm{c}}$, and Joseph S. Takahashi ${ }^{\mathrm{a}, \mathrm{b}, 2}$

${ }^{a}$ Department of Neuroscience, University of Texas Southwestern Medical Center, Dallas, TX 75390-9111

${ }^{b}$ Howard Hughes Medical Institute, University of Texas Southwestern Medical Center, Dallas, TX 75390-9111

${ }^{\mathrm{c} D}$ Department of Internal Medicine, Division of Hypothalamic Research, University of Texas Southwestern Medical Center, Dallas, TX 75390-9111

${ }^{1}$ Present Address: The Jackson Laboratory, Bar Harbor, ME 04609

\footnotetext{
${ }^{2}$ To whom correspondence should be addressed:

Dr. Joseph S. Takahashi

HHMI, Department of Neuroscience

University of Texas Southwestern Medical Center

5323 Harry Hines Blvd., NA4.118

Dallas, TX 75390-9111 USA
}

Tel: (214) 648-1876

Fax: (214) 648-1801

joseph.takahashi@utsouthwestern.edu

Classification: Biological Sciences - Genetics

Keywords: mutagenesis screen, fatigue, GLUT4, mouse model, sleep, voluntary wheelrunning activity 


\section{ABSTRACT}

2 In a screen of voluntary wheel-running behavior designed to identify genetic mouse

3 models of chronic fatigue in ENU mutagenized C57BL/6J mice, we discovered two lines

4 that showed aberrant wheel-running patterns. These lines both stem from a single

5 original founder identified as a low body-weight candidate in a recessive screen.

6 Progeny from both of these lines showed the abnormal wheel-running behavior, with

7 affected mice showing significantly lower daily activity levels than unaffected mice. They

8 also exhibited low amplitude circadian rhythms, consisting of lower activity levels during

9 the normal active phase, and increased levels of activity during the rest or light phase,

10 but only a modest alteration in free-running period. Their activity is not consolidated into

11 longer bouts, but is frequently interrupted with periods of inactivity throughout the dark

12 phase of the light-dark (LD) cycle. As seen with the low body weight, expression of the

13 behavioral phenotypes in offspring of strategic crosses was consistent with a recessive

14 heritance pattern. Mapping of these phenotypic abnormalities showed linkage to a

15 single locus on chromosome 11, and whole exome sequencing (WES) identified a

16 single point mutation in the SIc2a4 gene encoding the GLUT4 insulin-responsive

17 glucose transporter. The single nucleotide change ( $A$ to $T$ ) was found in the distal end of

18 exon 10, and results in a premature stop (Y440*). To our knowledge, this is the first time

19 a mutation in this gene has been shown to result in extensive changes in general

20 behavioral patterns.

\section{SIGNIFICANCE STATEMENT}

23 Chronic fatigue is a debilitating and devastating disorder with widespread consequences

24 for both the patient and the persons around them, but effective treatment strategies are

25 lacking. The identification of novel genetic mouse models of chronic fatigue may prove

26 invaluable for the study of its underlying physiological mechanisms and for the testing of

27 treatments and interventions. A novel mutation in Slc2a4 (GLUT4) was identified in a

28 forward mutagenesis screen because affected mice showed abnormal daily patterns

29 and levels of wheel running consistent with chronic fatigue. This new mouse model may

30 shed light on the pathophysiology of chronic fatigue. 


\section{INTRODUCTION}

32 Fatigue is a debilitating symptom comorbid with many disorders and disease states, and

33 may also present without readily identifiable associated disorders, the latter referred to

34 as idiopathic fatigue (1). Fatigue persisting 6 months or more is considered chronic

35 fatigue and if additional criteria are met, a diagnosis of Chronic Fatigue Syndrome (CFS)

36 can be made (2-4). The Centers for Disease Control and Prevention define chronic

37 fatigue syndrome as "a debilitating and complex disorder characterized by intense

38 fatigue that is not improved by bed rest and that may be worsened by physical activity or

39 mental exertion". Fatigue is associated with increased disability, impaired functioning

40 and increased mortality (5-7). Its negative impact on activity and overall quality of life

41 further increases the need for adequate and effective treatment, although this has

42 remained elusive.

43 The development of appropriate animal models to study the underlying

44 mechanisms of fatigue is crucial, but is hampered by the lack of a widely accepted

45 operational definition of fatigue $(8,9)$. Currently there are several different types of

46 mouse models, including those based on forced activity (e.g., treadmill or swimming),

47 neurodegenerative disorders (e.g., Parkinson's or Huntington's disease), cancer and its

48 treatments, or aging, but by far the most common types of models involve fatigue

49 following an immune challenge (e.g., those mimicking viral or parasitic infection) (10-

50 16). A commonality shared by all of these model systems is a resulting alteration of

51 behavioral output, such as voluntary wheel running (9).

$52 \quad$ The mouse is an ideal model organism to investigate physiological and

53 neurological pathways involved in fatigue $(9,17)$. Although the psychological and

54 emotional aspects of fatigue may be somewhat difficult to study in mice, the behavioral

55 components are more readily comparable. Models based on reduced or altered

56 voluntary wheel-running activity mimic the reduced voluntary physical activity exhibited

57 by humans with fatigue. Wheel running is a robust behavior in rodents, and is amenable

58 to quantitative analysis (17-19). Some characteristics that may imply fatigue in mice

59 when tested on running wheels include reduced daily amount of activity, changes in

60 pattern or day-night distribution, a lack of consolidated running with an increase in the 
61 number of distinct bouts, or a delayed onset or shortened duration of the daily active 62 phase.

63 There is some indication that there are genetic components related to the 64 development and manifestation of fatigue (20-23). Polymorphisms in several human 65 candidate genes (e.g., the adrenergic receptor a1 (ADRA1A), the serotonin transporter 66 (5-HTT or SLC6A4) or receptor (HTR2A), tyrosine hydroxylase (TH), corticosteroid67 binding globulin (CBG), corticotropin releasing hormone receptor 1 (CRHR1), the cytokine IL-1B, neuronal PAS domain protein 2 (NPAS2), the nuclear receptor subfamily 3; group C, member 1 glucocorticoid receptor (NR3C1), and the glutamate receptor ionotropic kinase 2 (GRIK2)) have been linked to both the occurrence and severity of fatigue symptomology (24-30). A study comparing chronic fatigue syndrome patients to

72 healthy controls using a single nucleotide polymorphism (SNP) chip with over 900,000

73 SNPs identified 442 candidates associated with chronic fatigue syndrome, and

74 highlighted two genes never before associated with fatigue (C-type lectin domain family

75 4, member M (CLEC4M) and coiled-coil domain containing 157 protein (CCDC157))

76 (31). In addition, mice with mutations in several genes have shown phenotypes consistent with fatigue (e.g., corticosteroid-binding globulin (CBG), recombinase activating gene 2 (RAG2), and interleukin-10 (IL-10)) (32-34).

We know that random mutagenesis paired with forward genetic screening has

80 proven invaluable in establishing and expanding understanding of gene function (3543). In the field of circadian rhythms, forward genetics has been a powerful tool for identifying both clock and clock-controlled genes (44). We systematically screen

83 randomly mutagenized mice on running wheels in search of lines exhibiting alterations

84 in voluntary wheel-running activity. We believe that the identification of mouse lines that 85 harbor mutations resulting in altered behavioral patterns consistent with chronic fatigue, and the subsequent identification of the responsible gene(s) or pathways, will contribute

87 significantly to our understanding of the pathophysiology of this debilitating disorder.

As part of a larger phenotype-driven ENU mouse mutagenesis project (45), we

89 screened mice for abnormalities in the expression of voluntary wheel-running activity,

90 with the hope of identifying genes involved in behavioral chronic fatigue. We describe 
91 the identification of two independent lines of mice stemming from a single mutagenized

92 founder showing profound alterations in amount, pattern and distribution of daily wheel

93 running resulting from a single point mutation (twiggy; MGl:5805978) in Slc2a4

94 encoding the insulin-responsive glucose transporter, GLUT4. To our knowledge, this is

95 the first description of impaired functioning of this gene resulting in extensive behavioral

96 abnormalities. The finding that a mutation in this gene results in widespread behavioral

97 modifications and altered expression of daily activity patterns indicates that its role is

98 likely more diverse than has previously been appreciated.

99

100

101

102

103

104

105

106

107

108

109

110

\section{RESULTS}

Mice show abnormal, low amplitude voluntary wheel-running activity patterns. In a voluntary wheel-running screen designed to isolate mice with heritable abnormal patterns of behavior, we identified two lines of mice showing abnormal activity consistent with chronic fatigue (Fig. 1). These two lines both stemmed from a single, third generation (G3) founder female identified as having low body weight (Fig. S1 A), which we named 'twiggy' and which was backcrossed to wildtype C57BL/6J to produce $\mathrm{N} 2$ progeny (Fig. S1 B). Two additional G3 pedigrees stemming from the same mutagenized founder (G0) male also contained affected G3 mice with low body weight (2 out of 8 mice and 3 out of 26 mice, respectively) but none of these G3s produced enough offspring for heritability testing. N2 progeny of the twiggy G3 founder female showed body weights within the normal range, but an intercross of N2 mice produced a subset ( 5-8 out of 57 ) that showed the low body-weight phenotype (Fig. S1B). A subsequent backcross of affected N2F2 mice to wildtype C57BL/6J produced N3 progeny that were unaffected, indicating that this phenotype is expressed in a non-sexlinked, recessive heritance pattern. Despite the low body-weight phenotype and overall smaller appearance, affected animals were completely healthy, and there was no observed effect on reproductive fitness.

Mice were maintained in two distinct lines following initial heritability testing (Fig. S1 C and D; Table S1). The first is a backcross/intercross (B/Int-x) line generated and maintained by backcrossing affected (presumably homozygous) mice to C57BL/6J, and 
121 then intercrossing the resulting offspring, producing alternating generations of obligate

122 heterozygous carrier "B/Int-x (N\#)" mice, and "B/Int-x (N\#F2)" mice of all 3 possible

123 genotypes $(+/+,+/ \mathrm{m}$, and $\mathrm{m} / \mathrm{m} ; \sim 25 \%, \sim 50 \%$ and $\sim 25 \%$, respectively; Fig. S1 C and D -

124 left panels; Table S1; where N\# denotes the number of backcross generations). The

125 second is an incross (I-x) line generated and maintained by crossing affected siblings

126 with each other over successive generations beginning with the first N2F2 progeny

127 shown in Fig. S1 B, to produce a homozygous line (Fig. S1 C and D - right panels). The

128 body weights of 8 sequential generations of each line are shown in Fig. S1 C (males)

129 and D (females), and, while all 8 I-x generations show body weights lower than the

130 screening mean, mice in the B/Int-x show a distinct generation-alternating pattern of

131 results.

132 Because of the low body weight, we measured fasting blood glucose from

133 individuals of both the B/Int-x and I-x lines. Although body weights following a $4 \mathrm{~h}$ fast

134 were significantly lower for both males and females of the I-x line than those of the B/Int-

$135 \mathrm{x}$ line (Fig. S1 E), there was no significant difference between the two lines in fasting

136 blood glucose (Fig. S1 F). Values for all mice tested were within the normal blood

137 glucose range for C57BL/6J mice reported by Jackson Labs (Center for Genome

138 Dynamics; CGDpheno1 glucose (plasma GLU, 4h fast); phenome.jax.org).

When mice stemming from these lines were housed in cages with free access to

140 a running wheel, a number of mice in the B/Int-x (N6F2) line showed a hypoactive and

141 disrupted pattern of voluntary wheel-running activity (Fig. 1 A and B). Representative

142 activity plots (actograms) of two mice of the B/Int-x (N6F2) line are shown, with one

143 exhibiting normal levels and patterns of activity (Fig. 1 A) and one showing the abnormal

144 phenotype (Fig. 1 B). In contrast, all the mice in the I-x (N2F10) line showed this same

145 aberrant pattern (Fig. $1 \mathrm{C}$ and D), consisting of low activity levels, increased bouts of

146 activity during the light or rest phase, non-consolidated or disrupted nocturnal activity,

147 and irregular onsets of the main nocturnal bout. When affected mice from the I-x

148 (N2F10) line were backcrossed to C57BL/6J mice in a test cross (T-x (N3); Table S1),

149 none of the progeny showed the phenotype, and circadian, or daily, wheel-running

150 behavior was indistinguishable from that of wildtype mice (Fig. $1 \mathrm{E}$ ). Average waveforms 
151 of 10 days of recording under a light-dark (LD) cycle clearly show that overall wheel-

152 running activity levels are significantly decreased in mice of the I-x (N2F10) line (Fig. 1

153 F). The B/Int-x (N6F2) line included some mice that showed the phenotype and some

154 that didn't, whereas none of the T-x (N3) mice were affected, and these showed a

155 normal circadian pattern of activity with high levels of nocturnal wheel running.

157 number of activity bouts per day (Fig. $1 \mathrm{I})$ recorded during the LD cycle, showed

158 significant differences among progeny of the three types of crosses, with mice of the I-x

159 (N2F10) line showing low activity, with higher levels of activity during the rest phase and

160 higher numbers of distinct activity bouts. Differences in activity levels were maintained

161 when mice were recorded in constant darkness (DD; Fig. $1 \mathrm{~J})$, and the amplitude of the

162 wheel running rhythm in affected mice was significantly lower than in unaffected mice

163 (Fig. $1 \mathrm{~K}$ ). The period of the free-running circadian rhythm recorded in DD was

164 significantly longer for mice of the I-x (N2F10) line (Fig. $1 \mathrm{~L}$ ), although these values fall

165 within the normal range for wildtype C57BL/6J (18).

167 Frequent rest bouts interrupt nocturnal wheel-running activity in affected mice.

168 In order to characterize further the abnormal behavioral pattern these mice exhibit, we

169 assessed their behavioral profiles during the dark phase using both time-lapsed

170 photography (Fig. 2) and video recording (Fig. 3). Images captured once every min for

171 15h beginning at the start of the dark phase for 3 individual wildtype C57BL/6J (Fig. S2)

172 and 5 individual I-X (N2F14) mice (Fig. S3) were scored on a range of 9 behavioral

173 states. The I-X (N2F14) mice were each recorded twice, with one week occurring

174 between recording sessions (Fig. S3). The ethogram of scored behaviors for the

175 representative wildtype C57BL/6J mouse shows extended bouts of wheel running with

176 short interruptions for drinking, eating, and general cage activity (Fig. 2 A). This mouse

177 only adopted a body posture consistent with sleep during the end of the end of the dark

178 phase (i.e., after 3:00 h). The amount of time spent in the various behaviors for the first

$1796 \mathrm{~h}$ of the dark phase $(18: 00-0: 00 \mathrm{~h})$ are represented in a pie chart for this individual

180 mouse in Fig. $2 \mathrm{C}$ and averaged for all 3 wildtype mice in Fig. 2 D. The pattern of 
181 behaviors shown by the representative I-x (N2F14) mouse (Fig. 2 B) does not resemble

182 that recorded for wildtype mice. Consolidated, extended bouts of wheel running are

183 absent, and running is interrupted with periods of time when the mouse is near the

184 wheel, but is not active. During these periods of time, the mouse is seen to adopt a low,

185 crouched posture indicative of sleep (Fig. $2 \mathrm{G}$ - right panel). The distribution of

186 behaviors for the first $6 \mathrm{~h}$ of the dark phase $(18: 00-0: 00 \mathrm{~h})$ for this mouse is shown in

187 Fig. 2 E, and for all 5 I-x (N2F14/15) mice recorded twice in Fig. 2 F. Most notably, 188 running is significantly decreased for these mice compared to wildtype (Fig. 2 C-F),

189 Even more surprising, all 5 I-X (N2F14/15) mice show sleep postures during this 6h time 190 period on both recording sessions, whereas wildtype mice were never observed to do 191 this (Fig. S2 and S3).

192 Videos recorded during the first few hours of the dark phase for three individual 193 mice of the I-x (N2F21) and T-x (N11F3) generations were scored and the data are 194 plotted along with those for two unaffected T-x (N3F2) mice (Fig. 3 A and B). Mice of

195 both affected groups showed extremely low wheel-running levels compared to T-x 196 (N3F2) unaffected mice (Fig. 3 A and B - upper panels). The T-x (N3F2) mice never 197 showed behavioral arrest and lowered posture consistent with sleep during this time of 198 the day, whereas all three individuals of the other two lines did (Fig. 3 A and B - lower 199 panels).

201 Abnormal phenotypes map to a single locus on chromosome 11.

202 To map the mutation, we crossed affected mice from both the I-x and the B/Int-x lines to 203 C57BL/10J mice, and then intercrossed the resulting $M-x(F 1)$ progeny $(n=92)$ to 204 produce M-x (F2) mice (Table S1). In total, 207 M-x (F2) mice from 9 independent 205 matings were produced and were tested on running wheels under LD and DD conditions 206 for the presence of the behavioral phenotype (Fig. 4). All data were compared to F1 and 207 F2 mice generated from crosses of wildtype C57BL/6J and C57BL/10J mice 208 (designated as WTB6B10F1 $(n=63)$ and WTB6B10F2 $(n=112)$, respectively).

209 Representative actograms recorded from two M-x (F2) mice showing the low-amplitude, 210 low activity, highly disrupted behavioral wheel-running phenotype are shown in Fig. 4 B 
211 and $\mathrm{C}$, with an unaffected mouse shown in Fig. 4 A. No WTB6B10F1, WTB6B10F2 or

$212 \mathrm{M}-\mathrm{x}(\mathrm{F} 1)$ mouse exhibited this constellation of behavioral phenotypes, although some

213 individuals did show some of its component characteristics (e.g., low activity but no

214 increased running in the light phase or no lack of consolidation of nocturnal activity).

215 Mice of the M-x (F2) generation showed decreased activity recorded under LD (Fig. 4D)

216 and DD (Fig. 4E), increased amount of activity in the light phase (Fig. S4 A), and a

217 lower amplitude rhythm recorded in DD (Fig. 4F). There was no significant difference

218 found in number of activity bouts per day (Fig. S4 B), and the period of the free-running

219 rhythm was not altered for M-x (F2) mice, although interestingly, the WTB6B10F2 mice

220 did show a significantly shorter period (Fig. S4 C).

221 Using a genome-wide SNP panel of markers designed to differentiate C57BL/6J

222 from C57BL/10J (Table S2), the genotypes of 74 selected affected and unaffected M-x

223 (F2) mice were determined, and were assessed for linkage to the various phenotypic

224 measurements. Significant log odds ratio (LOD) peaks with linkage to a single locus on

225 chromosome 11 were found for the amount of activity recorded in LD (Fig. 4 G) and DD

226 (Fig. $4 \mathrm{H}$ ), and amplitude of the rhythm in DD (Fig. 4 I). No significant linkage was found

227 for the percentage of activity occurring during the light phase (Fig. S4 D and G), the

228 number of activity bouts occurring per day (Fig. S4 E and H), or the free-running period

229 in DD (Fig. S4 F and I). Activity levels in LD (Fig. $4 \mathrm{~J}$ ), and in DD (Fig. 4 K), as well as

230 the amplitude of the rhythm in DD (Fig. $4 \mathrm{~L}$ ), are plotted as a function of genotype at

231 SNP marker rs13481117 located on chromosome 11, position 79252230. Mice

232 homozygous for the C57BL/6J allele (designated as "B6/B6") show lower activity levels

233 in both LD and DD with a lower amplitude circadian rhythm.

235 A point mutation in Slc2a4 encoding GLUT4 is identified in affected mice.

236 In order to identify the gene responsible for the abnormal phenotypes described, we

237 performed whole-exome sequencing (WES) of pooled DNA collected from mice of the I-

$238 \times($ N2F20) generation, and those from a test cross stemming from affected B/Int-x

239 (N11F2) mice (T-x (N11F3); N=16 for each line). Representative activity records of mice

240 in the various generations used for this experiment are shown in Fig. S5 A-F. Body- 
241 weight data collected at 8 weeks of age for both male and female mice in the $1-x$

242 (N2F20) and T-x (N11F3) generations, as well as for those of the B/Int-x (N11) and

243 B/Int-x (N1F2) used to produce the T-x (N11F3) mice, reveal that mice of both lines

244 chosen for WES have significantly lower body weight (Fig. S5 G). In addition, mice of

245 both presumptive homozygous lines (I-x (N2F20) and T-x (N11F3)) showed significantly

246 lower levels of activity in LD compared to mice from the B/Int-x (F1) and B/Int-x (F2)

247 generations (Fig. S5 H). Importantly, activity levels and wheel running patterns did not

248 differ between these two presumptive homozygous lines, indicating that both were, in

249 fact, homozygous for the same genetic mutation.

250 WES results revealed a single point mutation in the Slc2a4 gene encoding

251 GLUT4, the insulin-responsive glucose transporter (Fig. 5). No other gene within the

252 linked region of chromosome 11 had polymorphisms represented in both of the samples

253 analyzed. Remarkably, all reads covering this region of the genome for both of the

254 pooled samples showed this same SNP (Fig. 5 A - red arrows). For clarity, 19

255 representative reads from each of the pooled samples are shown at higher

256 magnification (Fig. $5 \mathrm{~A}$ - lower panel). A single nucleotide substitution (A to T) within the

257 distal end of exon 10 of the gene results in a premature stop (Y440*; see Fig. 5 B).

258 Genotyping for this SNP revealed that all mice in the 2 test cross lines were

259 homozygous for the mutant allele of this gene, whereas mice in the B/Int-x lines showed

260 the expected Mendelian ratio of genotypes at this mutant locus. Surprisingly, western

261 blot analysis showed that homozygous mice had higher levels of protein in the

262 gastrocnemius muscle than heterozygotes and wildtype littermates (Fig. 5 C), indicating

263 that the mutation appears to result in protein accumulation or increased expression.

264 Despite the higher levels of accumulated protein, mice homozygous for the

265 twiggy mutation show phenotypes similar to those shown by GLUT4 knockout mice (46-

266 50). Despite normal fasting glucose levels (Fig. 6 B and also Fig. S1 F), Slc2a4 ${ }^{\text {twgy/twgy }}$

267 mice had impaired glucose clearance with significantly elevated blood glucose levels 15,

26830 and 45 min after glucose injection (Fig. 6 A). Both +/twgy and twgy/twgy male mice

269 showed decreased body weight, although at this age female body weights did not differ

270 significantly among genotypes (Fig. 6 C). Like GLUT4 knockout mice (46), twgy/twgy of 
271 both sexes had enlarged hearts (Fig.6 D). The twiggy mutation in Slc2a4 resulted in

272 impaired exercise tolerance, with twgy/twgy mice showing shorter times (Fig. 6 E) and

273 distance (Fig. 6 F) to exhaustion upon testing on a treadmill. Homozygous twgy/twgy

274 mice also had elevated blood glucose (Fig. 6 G) and decreased lactate (Fig. 6 H) levels

275 following completion of the treadmill task.

276 When mice of the B/Int-x (N12F2) line were housed with access to a running

277 wheel, only those mice with the homozygous twgy/twgy genotype showed the

278 behavioral pattern identified in the original two lines (Fig. $7 \mathrm{~A}$ and Fig. 1 A-D). Thus, this

279 pattern, consisting of decreased wheel-running behavior (Fig. 7 B) with a characteristic

280 non-consolidated, messy pattern of expression, an increase in activity in the light phase

281 (Fig. 7 C) and increased number of shorter, distinct bouts of activity, that is indicative of

282 an overall excessive fatigability and sleep/wake disruption, shows a clear recessive

283 mode of inheritance.

\section{SUMMARY AND DISCUSSION}

286 Excessive fatigue that defies simple explanation affects large subsections of the

287 population at one time or another, and results in diminished daily functioning and quality

288 of life $(1,3,4)$. It can be a symptom or side effect of varied diseases and disorders and 289 their treatments, but can also be manifest independent of other comorbid diagnoses or 290 etiologies (51-53). For those persons suffering from this debilitating disorder, and their

291 loved ones, effective treatments would be most welcome, but in order for new

292 treatments to be identified and developed, informative animal models of chronic fatigue

293 are crucial. We carried out a forward genetic screen in order to identify mouse lines

294 showing behavioral characteristic of chronic fatigue. We describe the identification of a

295 novel mutation in the S/c2a4 gene encoding GLUT4 that results in a distinct daily

296 voluntary wheel-running pattern that is consistent with chronic fatigue. Affected mice

297 homozygous for the twiggy mutation of Slc2a4 show a behavioral pattern consisting of

298 low, non-consolidated and messy wheel-running activity, with increased short bursts of

299 activity interrupting the rest phase. These mice sleep during times of the day that normal

300 nocturnal wildtype mice would never sleep (i.e., during the first half of the active phase). 
301 This pattern of behavior is consistent with increased fatigability, and suggests that

302 impaired functioning of the insulin-responsive glucose transporter (GLUT4), or other

303 impairments in glucose regulation and homeostasis may play a role in the underlying

304 pathophysiology of chronic fatigue.

305 Additional phenotypic characteristics observed in Slc2a4 ${ }^{\text {twgy/twgy }}$ homozygous

306 mice are consistent with a non-functioning GLUT4 transporter (46). These

307 characteristics include a low body weight, impaired glucose tolerance response with a

308 normal baseline fasting blood glucose level, increased heart mass consistent with

309 cardiac hypertrophy, decreased exercise tolerance, and increased fatiguing response

310 with abnormal blood glucose and lactate levels following exercise. Surprisingly, although

311 these phenotypic characteristics largely match those described for the GLUT4 null

312 mouse $(46-49,54-56)$, the protein levels in Slc2a $4^{\text {twgy/twgy }}$ homozygous mice were found

313 to be elevated, suggestive of protein accumulation due to impairments in turnover or

314 degradation of the protein, or compensatory upregulation. It is possible that the mutation

315 results in a malformation of GLUT4 that interferes with its translocation or fusion to the

316 plasma membrane, or that it impairs its function as a glucose transporter. Either of these

317 possibilities may result in a compensatory over production of the protein. The premature

318 stop introduced into exon 10 of the gene would likely result in the truncation of the

319 protein and absence of the carboxy terminus. There are several important regulatory

320 elements located in this region of the protein that play a role in its subcellular

321 localization and transmission to the plasma membrane in response to circulating insulin,

322 dietary components and metabolites, or exercise-related signaling pathways (57-62). It

323 remains to be determined whether the phenotypes resulting from the presumably

324 truncated form of Slc2a4 are the result of impaired functioning of the glucose transporter

325 itself, or from impairments in its vesicular localization and trafficking to the membrane in

326 response to insulin or other signaling cascades.

$327 \quad$ The constellation of phenotypes described for mice in the twiggy lines, including

328 low body weight, abnormal voluntary wheel running, and physiological alterations such

329 as enlarged hearts and impaired glucose tolerance responses, all show a clear

330 recessive heritance pattern. For virtually all phenotypes measured, heterozygous 
Slc2a $4^{+/ t w g y}$ mice were indistinguishable from their wildtype littermates and/or wildtype C57BL/6J controls. This is in sharp contrast to the GLUT4 knockout heterozygous

333 mouse that exhibits phenotypes consistent with diabetes (63-66). It has been suggested

334 that this diabetic phenotype, which is manifest in only a subpopulation of the

335 heterozygous knockouts and appears to vary with age, could be an artifact of genetic

336 background; however, this remains an open issue (64). In addition, GLUT4 protein

337 levels are diminished in heterozygous GLUT4 knockout mice (63-66). In contrast, 338 protein levels of GLUT4 were unchanged in the muscle of Slc2a ${ }^{+/ t w g y}$ mice. They were

339 not diminished as in the heterozygous knockout, absent as in the homozygous

340 knockout, nor elevated as in the S/c2a $4^{\text {twgy/twgy }}$ mice. This finding reinforces the idea that

341 this mutation resulting in a premature stop in exon 10 of the gene does not simply knock

342 out GLUT4, and therefore it represents a novel model that can be used to further

343 characterize GLUT4 functioning.

$344 \quad$ The GLUT4 glucose transporter was historically considered a peripheral-acting

345 glucose channel, with expression thought to be limited to muscle, adipose tissues, and

346 heart $(67,68)$. Recent studies have shown that it is, in fact, expressed more widely and

347 can also be found in distinct neuronal populations throughout the brain in mice (69).

348 This more varied expression pattern and anatomical localization suggests that GLUT4

349 functions are more complex than simply a shunt for glucose in peripheral tissues. For

350 example, a very recent study shows that GLUT4 functions to maintain neuronal, and

351 specifically synaptic, energy requirements and is essential for maintaining extended

352 synaptic transmission (70). The use of conditional genetic tools has also shed more light

353 on the diverse functions of GLUT4. Interfering with insulin signaling specifically in all

354 tissues expressing GLUT4 results in a diabetic phenotype that is due, at least in part, to

355 impaired insulin signaling in neuronal populations (71). It is interesting to note that these

356 mice showed decreased body weight, but did not show any alterations in activity

357 amounts or patterns (71). Mice with GLUT4 removed selectively from the brain show

358 that neuronal GLUT4 is critical for assessing and reacting to changes in blood glucose

359 levels (72); however, it is possible that some of these effects are non-specific Cre-driver

360 related since Nestin-Cre is expressed peripherally and mice carrying this transgene 
361 have been shown to have metabolic phenotypes (73). Work has been initiated to try to 362 identify the function of distinct subgroups of GLUT4 neurons using methods to isolate 363 and/or selectively ablate those expressing this gene $(74,75)$. The removal of

364 hypothalamic neurons expressing GLUT4 resulted in metabolic changes that are consistent with impaired energy utilization and homeostatic processes, but did not result in locomotor deficits (75). These mice ate less and lost weight, had altered oxygen consumption and energy expenditure, and increased fasting blood glucose and aberrant glucose tolerance responses, but the amount and daily pattern of activity remained unchanged. These mice did, however, show a decreased activity response to an overnight fast, and did not increase activity levels as much as unablated controls (75).

371 Clearly, the use of these genetic tools to conditionally manipulate the expression of

372 GLUT4 in anatomically and temporally controlled ways will be critical in fully

373 characterizing the function of this glucose transporter. This should allow for the

374 determination of exactly how GLUT4 removal or altered function results in the seemingly 375 varied array of phenotypic outcomes described in the literature to date.

The identification of a novel mutation in the Slc2a4 gene resulting in a

377 constellation of abnormal behavioral characteristics consistent with chronic fatigue was

378 somewhat surprising, but not necessarily unexpected. It seems logical that impaired 379 glucose processing and homeostasis, with a concomitant altered tolerance of exercise, 380 would result in aberrant patterns of voluntary wheel-running activity as well. Voluntary 381 wheel running is increased in mice overexpressing GLUT4 in muscle (76), but GLUT4 382 expression levels did not differ between active and inactive mouse strains (77). It is 383 likely, however, that impaired glucose transport and cellular utilization would have some 384 effect on voluntary sustained activity. Switching from predominantly glucose to fatty acid 385 fuel utilization in muscle, either by transgenic or pharmacologic manipulation of the 386 peroxisome proliferator-activated receptor delta (PPARס) pathway, increases exercise 387 endurance in mice (78-80). Whether or not this increase in exercise capacity translates 388 into an increase in voluntary activity remains to be fully elucidated. For instance, mice 389 deficient in both cryptochrome 1 and 2 (Cry $1 / 2 \mathrm{dKO})$ ran faster and for longer distances 390 than wildtype or single knockout controls on a treadmill task, presumably through de- 
391 repression of PPAR activity; however, these mice showed decreased daily voluntary

392 wheel-running behavior (81). It remains to be determined whether or not treatment of

393 Slc2a ${ }^{\text {twgy/twgy }}$ mice with exercise mimetics would normalize some of the behavioral

394 abnormalities in wheel running that we observed (82). This may prove informative to the

395 investigation of how these novel pharmacological treatments may apply to patients with

396 chronic fatigue, for whom voluntary exercise programs have been less than successful.

397 Fatigue, and alterations in the activity of daily living are well-described side

398 effects and symptoms of both type I and type II diabetes (83-85). Like fatigue in other

399 patient populations, fatigue in diabetes is associated with physiological, psychological

400 and lifestyle variables, but may be exacerbated by fluctuations in blood glucose levels

401 and homeostasis. There is also some evidence suggestive of abnormal metabolic

402 responses in chronically fatigued patients, with abnormalities in energy and sugar

403 metabolism comprising one of the three main categories of pathways identified (86).

404 The identification of abnormalities in metabolic pathways in chronic fatigue, both as

405 candidates for geared treatment options, and as potential biological markers that can be

406 used to differentially diagnose various subtypes of the disorder, have received an

407 increase in scientific focus recently $(87,88)$.

$408 \quad$ Using an unbiased, forward genetic ENU mutagenesis screen for chronic fatigue, 409 we identified mice with a mutated form of the GLUT4 transporter; however, we are by no 410 means suggesting that mutations in GLUT4, or impaired glucose transport underlie all

411 manifestations of fatigue, although it may play a role in at least a subset of cases.

412 Rather, it is likely that chronic fatigue represents a persistent altered homeostatic state

413 in response to a variety of triggers and physiological adaptations (87). It is hoped that

414 additional screening of mutagenized mouse lines for abnormalities in the expression of

415 voluntary wheel-running behavior will yield additional candidate genes and pathways

416 that are implemented in this complex disorder. In the mean time, studies to determine

417 the effectiveness of treatment strategies geared towards normalizing glucose

418 processing and transport in the Slc2a $4^{\text {twiggy }}$ mice and other mouse models of chronic

419 fatigue may hold promise. In addition, assessing glucose tolerance in patients for whom

420 fatigue remains an unexplained symptom may be warranted. 


\section{MATERIALS AND METHODS}

\section{Animals and Housing}

424 All mice were group housed in standard mouse cages under a 12:12 LD cycle (lights on 425 at 6:00 AM) with water and regular mouse chow (2018 Teklad Global 18\% Protein

426 Rodent Diet, Envigo, Madison, WI, USA) available ad libitum, unless otherwise stated.

427 Mice were produced in our colony from strategic crosses, or were ordered from an in-

428 house breeding core facility (Mouse Breeding Core, Wakeland lab, UT Southwestern

429 Medical Center, Dallas, TX, USA), which orders its breeding mice from Jackson Labs

430 (Bar Harbor, ME, USA). Some wildtype C57BL/6J mice were also ordered from Jackson

431 Labs (Stock Number 000664) directly. The generation of the original ENU mutagenized

432 screening population and body weight screen were done at Northwestern University,

433 and all behavioral and subsequent phenotypic characterizations were performed at the

434 University of Texas Southwestern Medical Center. The Institutional Animal Care and

435 Use Committee (IACUC) of Northwestern University and the University of Texas

436 Southwestern Medical Center approved all animal procedures (ACUC\# 2003-0034, and

437 APNs 2009-0054, 2015-100925 and 2015-101140, respectively). The Slc2a4 ${ }^{\text {twiggy }}$ line

438 has been donated to Jackson Labs (C57BL/6J-Slc2a4 ${ }^{\text {twiggy } / J, ~ S t o c k ~ N u m b e r ~ 029699) . ~}$

440 ENU mutagenesis and Phenotypic Screening

441 The generation of N-Ethyl-N-nitrosourea (ENU; Sigma; catalog \#N3385) mutagenized

442 mice and their progeny for initial high-throughput screening was described previously 443 (45). Briefly, male C57BL/6J mice ( 6 weeks of age) were injected with $250 \mathrm{mg} / \mathrm{kg}$ of

444 ENU. Following a recovery period of $\sim 6$ weeks, these mice were crossed with wildtype 445 C57BL/6J females to produce generation 1 (G1) males which were again crossed to 446 wildtype C57BL/6J females to produce G2 females. These G2 females were 447 backcrossed to their G1 fathers to produce G3 mice of both sexes used for phenotype 448 screening.

$449 \quad$ More advanced generations of mice, further removed from the original G0 450 founders, were used for the more in depth behavioral phenotypic screening. Progeny of 
selected lines of mice that were at least 8 weeks old were individually housed in standard polycarbonate mouse cages (Fischer Scientific; catalog \# 01-288-1B and 01288-21) containing a 4.75" diameter stainless steel running wheel placed inside isolation cabinets containing 12 to 24 cages each, as described previously (19). Temperature and humidity were monitored throughout, and the mice were initially recorded under a 12:12 LD cycle (green LEDs, 100 lux at the level of the cage floor). Following the initial 3 weeks of testing under 12:12 LD, selected individual progeny from specific crosses were tested under constant darkness (DD) or constant light (LL) conditions. Wheelrunning behavior was recorded using ClockLab Data Acquisition Software (Actimetrics

460 Inc., Wilmette, IL, USA). Wheel-running data, specifically total daily amount, number of

461 daily bouts, and percentage of activity occurring in the light during LD recording, as well

462 as total daily amount of activity, free-running period, and amplitude of the rhythm 463 recorded in DD, were quantified using ClockLab Analysis Software (Actimetrics Inc., 464 Wilmette, IL, USA). For all data quantification, recording days 6-15 under LD and 31-40 under DD were used. All wheel-running cages, food and water bottles were changed every 21 days.

Behavioral Time-Lapsed Photography and Video Recording

I-x (N2F14 and N2F15) and C57BL/6J wildtype mice were housed in individual runningwheel cages as described above. Using a Sony Handycam DCR-HC62 camera with an LED IR light source, photographs were taken at 1 min intervals beginning at lights off (or 18:00) for $15 \mathrm{~h}$. The resulting pictures were scored from 1 to 9 for the behavioral state or cage location depicted in each. The scores used were: 1) drinking, 2) eating, 3) near the

474 food hopper, 4) on the wheel running, 5) near the wheel awake, 6) end of the cage 475 awake, 7) end of the cage resting, 8) near the wheel asleep, and 9) end of the cage 476 asleep. These scores were plotted in ethograms according to the time at which each 477 picture was taken.

$478 \quad$ Video recording of $3 \mathrm{I-x}$ (N2F21) mice, and mice from two different test crosses

479 (T-x (N3F2) $n=2$ and T-x (N11F3) $n=3)$, was done under the same IR light source 480 described above using the same Sony Handycam camera with Sony Premium Mini DV 
481 Cassettes (DVM60 ME LP:90) from 20:00 to 21:30 (i.e., 2h to 3.5h into the dark phase).

482 Wheel running was recorded throughout this period as described above. The resulting

483 video was scored for behavioral arrest indicative of sleep by two researchers blind to

484 mouse identity. Periods of immobility with lowered posture lasting longer than 40

485 consecutive seconds were scored as sleep (89).

487 Body Weight, Fasting Blood Glucose, Health Assessment, and Tissue Collections

488 All mice produced in our colony were weighed at specific ages through development. At

489 weaning, and at regular intervals thereafter, mice were visually inspected for general

490 health and wellness. For the determination of blood glucose levels, mice were fasted for

$4914 \mathrm{~h}$ during the middle of the light phase (a time when mice do not normally eat). Body

492 weights were measured at the beginning and the end of the $4 \mathrm{~h}$ fast, and blood glucose

493 was determined from a small droplet of blood taken from the tip of the tail, using the

494 Precision Xtra blood glucose meters and glucose test strips (Abbott Diabetes Care Inc.,

495 Almeda, CA, USA). At the end of experiments and phenotypic characterization, mice

496 were euthanized with $\mathrm{CO}_{2}$ followed by cervical dislocation. In some cases, tissues

497 (tailtips, spleens and hearts) were collected for weighing or DNA extraction followed by

498 genotyping.

$500 \quad$ Mapping

501 Affected mice from the I-x and B/Int-x lines were selected and crossed with wildtype

502 C57BL/10J mice (Stock Number 000665, Jackson Labs, Bar Harbor, ME, USA) to

503 produce mapping (M-x) F1 mice that were intercrossed to produce $\mathrm{F} 2$ progeny.

504 Additional crosses of wildtype C57BL/6J and C57BL/10J were also set up to produce

505 control (WTB6B10) F1 and F2 mice. All mice produced were individually housed in

506 running-wheel cages after 8 weeks of age and their behavior was recorded in LD (for 3

507 weeks) and DD (for 3 weeks) as described above. Following behavioral testing, mice

508 were euthanized and spleens were collected, frozen onto dry ice, and then stored at -

$50980^{\circ} \mathrm{C}$. Spleen DNA from 74 selected mice (half presumptive homozygous mutants and

510 half presumptive wildtypes or heterozygotes) was extracted using phenol chloroform 
511 genomic DNA extraction method. The final DNA pellet was suspended in RNAse free

512 H2O. Concentrations were determined with a NanoDrop spectrophotometer, and DNA

513 was genotyped using Taqman probes on Fluidigm platform using the 96.96 chip

514 according to manufacturer instructions as described previously (90). QTL linkage

515 analysis was performed using R/QTL as described previously (90, 91). Genotyping

516 failed for one spleen sample, and these data were subsequently removed from further

517 analyses.

519 Whole Exome Sequencing

520 Test crosses (T-x) of affected, presumably homozygous B/Int-x (N11F2) were set up to 521 produce homozygous T-x (N11F3) progeny. Following phenotype recording on wheels, 522 mice were euthanized and spleens were collected from 16 representative T-x (N11F3)

523 individuals and 16 representative l-x (N2F20) mice. Spleens were quickly frozen on dry 524 ice and were then stored at $-80^{\circ} \mathrm{C}$ until DNA was extracted using the Gentra Puregene 525 Mouse Tail/Tissue Kit (Catalog number 158906 - Cell Lysis Solution, 158918 -

526 Puregene Proteinase K, 158922 - RNase A Solution, and 158910 - Protein Precipitation

527 Solution; Qiagen, Germantown, MD, USA) according to manufacturer instructions. DNA 528 was dissolved in sterile RNAse free $\mathrm{H} 2 \mathrm{O}$ and concentrations were determined using a 529 NanoDrop spectrophotometer (ND-2000, ThermoFisher Scientific, Waltham, MA, USA)

530 and Qubit 2.0 fluorometric quantitation (Q32866, ThermoFisher Scientific, Waltham, MA, 531 USA). DNA samples for the 16 individuals of each of the two lines were pooled into two 532 samples that were used for whole exome sequencing.

533 Whole-exome capture, sequencing, and analysis were performed by the UT

534 Southwestern McDermott Center Next-Generation Sequencing and Bioinformatics Core.

535 After initial DNA quality assessment on a 1.8\% agarose gel and concentration

536 quantification using a Qubit 3.0 Fluorometer (Invitrogen, Carlsbad, CA USA), fragment

537 libraries were prepared using SureSelectXT2 HSQ Reagent Kit (Agilent Technologies,

538 Inc., Santa Clara, CA, USA). Samples were sheared on the Covaris S-2 sonicator

539 (Covaris, Woburn, MA, USA) and end-repaired, after which the 3' ends were adenylated 540 and barcoded with pre-capture indexing adapters. Following amplification and 
541 purification, the sizes of the fragment libraries were assessed on the Agilent 2100

542 BioAnalyzer (Agilent Technologies, Inc., Santa Clara, CA, USA) and concentrations

543 were once again determined by Qubit fluorometric quantitation. Samples were pooled in

544 equimolar amounts and captured with the SureSelectXT2 Target Enrichment System for

545 Mouse (Agilent Technologies, Inc., Santa Clara, CA, USA), followed by a final Qubit

546 concentration quantification, and were run on the Illumina NextSeq 500 platform

547 (Illumina Inc., San Diego, CA, USA) using 150PE SBS v2 chemistry. Sequence reads

548 were mapped to the Genome Reference Consortium GRCm38 reference genomic

549 sequence for C57BL/6J using BWA (92). Both samples resulted in mean coverage

550 greater than 120X. Variants were called using the Genome Analysis ToolKit (GATK)

551 (93), and annotated using snpEff (94). Reads were visualized and plotted using the

552 Broad Institute Integrative Genomics Viewer (IGV) to detect homozygous mutations

553 present in both samples.

555 Genotyping

$556 \mathrm{NaOH}$-extracted DNA from tailtip samples taken at weaning from progeny of selected

557 crosses was genotyped for the presence of the twiggy mutation with real-time PCR to

558 detect the SNP using the following primers: Slc2a4 Forward (ccagagaccacctacatggc)

559 Slc2a4 WTReverse (GTCGGCATGGGTTTCCAGaAT) and Slc2a4 MutantReverse

560 (GTCGGCATGGGTTTCCAGaAa). PCR reactions were done in a $10 \mu \mathrm{l}$ volume using

$561 \sim 10 \mathrm{ng}$ genomic DNA template with KAPA SYBR Fast ABI Prism 2X qPCR Master Mix

562 (KK4617; KAPA Biosystems, Wilmington, MA, USA), on an ABI 7900HT Fast Real-Time

563 PCR Machine (Applied Biosystem, Inc., Foster City, CA, USA) using MicroAmp 384-well

564 Optical Reaction Plates (Applied Biosystems, Inc., Foster City, CA, USA) with the

565 following thermo-cycling conditions: $95^{\circ} \mathrm{C}$ for 3 min followed by 40 cycles of $95^{\circ} \mathrm{C}$ for 3

$566 \mathrm{sec}$ and $62^{\circ} \mathrm{C}$ for $20 \mathrm{sec}$.

568 Glucose Tolerance Test

569 Progeny of select crosses were fasted for $4 \mathrm{~h}$ during the middle of the light phase (a time 570 when mice do not normally eat). Body weights were measured at the beginning and the 
571

572

573

574

575

576

577

578

579

580

581

582

583

584

585

586

587

588

end of the $4 \mathrm{~h}$ fast, and initial blood glucose was determined from a small droplet of blood taken from the tip of the tail using the Precision Xtra blood glucose meters and glucose test strips (Abbott Diabetes Care Inc., Almeda, CA, USA). Mice were then given an I.P. injection of $10 \%$ sterile glucose solution (1 g/kg; Sigma-Aldrich, St. Louis, MO, USA), and blood glucose values were measured again at 15, 30, 45, 60 and $120 \mathrm{~min}$ post injection.

\section{Endurance Treadmill Test}

To test exercise endurance capacity, mice of both sexes from selected crosses were run on a progressive treadmill running protocol. The mice were acclimatized to the motorized treadmill (Animal Treadmill Exer-3/6, Columbus Instruments, Columbus, $\mathrm{OH}$, USA) for two days prior to the endurance test. Day 1 of acclimatization lasted $20 \mathrm{~min}$ consisting of $5 \mathrm{~min}$ on the treadmill at rest $(0 \mathrm{~m} / \mathrm{min}), 5 \mathrm{~min}$ at $8 \mathrm{~m} / \mathrm{min}, 5 \mathrm{~min}$ at 10 $\mathrm{m} / \mathrm{min}$, followed by $5 \mathrm{~min}$ of rest $(0 \mathrm{~m} / \mathrm{min})$. Day 2 also lasted $20 \mathrm{~min}$ and consisted of 5 min on the treadmill at rest $(0 \mathrm{~m} / \mathrm{min}), 5 \mathrm{~min}$ at $10 \mathrm{~m} / \mathrm{min}, 5 \mathrm{~min}$ at $12 \mathrm{~m} / \mathrm{min}$, followed by $5 \mathrm{~min}$ of rest $(0 \mathrm{~m} / \mathrm{min})$. On day 3 , mice performed the endurance test to exhaustion, defined as when the mouse spent more than $5 \mathrm{sec}$ on the shock grid $(0.1 \mathrm{~mA} \times 163 \mathrm{~V}$ and $1 \mathrm{~Hz}$ ) without re-engaging with the treadmill, as described previously (95). The mice ran at a speed of $10 \mathrm{~m} / \mathrm{min}$ for the first $40 \mathrm{~min}$, then the rate was increased at $1 \mathrm{~m} / \mathrm{min}$ every $10 \mathrm{~min}$ until the speed reached $13 \mathrm{~m} / \mathrm{min}$, at which point the speed was increased at a rate of $1 \mathrm{~m} / \mathrm{min}$ every $5 \mathrm{~min}$ until exhaustion. Mice were then removed from the treadmill and blood glucose and lactate were immediately measured from tail blood using hand-held analyzers for glucose (Bayer Contour Blood Glucose Meter, Ascensia Diabetes Care US, Inc., Parsippany, NJ, USA) and lactate (Nova Biomedical Lactate Plus Meter, Waltham, MA, USA). Mice were euthanized by decapitation, and muscles were rapidly dissected and frozen on dry ice. All tissues were stored at $-80^{\circ} \mathrm{C}$ until used.

\section{Western Blotting}

Frozen gastrocnemius muscles collected from mice following the treadmill study were homogenized in ice-cold lysis buffer composed of T-PER ${ }^{\circledR}$ buffer (\#78510, 
601 ThermoFisher Scientific, Waltham, MA, USA), 1\% (v/v) of protease inhibitors cocktail

602 (P8340-5ML, Sigma-Aldrich, St. Louis, MO, USA), and phosphatase inhibitors cocktail 2

603 and 3 (P5726-5ML and P0044-5ML, Sigma-Aldrich, St. Louis, MO, USA). Samples were

604 homogenized using TissueLyser II homogenizer (Qiagen Inc., Valencia, CA, USA).

605 Homogenates were then solubilized by constant rotation for $1 \mathrm{~h}$ at $4^{\circ} \mathrm{C}$, and $10 \mathrm{~min} \mathrm{x}$

$6061,000 \mathrm{~g}$ at $4^{\circ} \mathrm{C}$. Protein concentration of the supernatant was determined via the Pierce

607 BCA protein assay kit (\#23225, ThermoFisher Scientific, Waltham, MA, USA). Samples

608 were then stored at $-80^{\circ} \mathrm{C}$ until further analysis. Equal amounts of total protein $(20 \mu \mathrm{g})$

609 per sample were diluted with appropriate volume of laemmle sample buffer (2X

610 concentrated; 4\% SDS, 10\% 2-mercaptoethanol, 20\% glycerol, 0.004\% bromophenol

611 blue and $0.125 \mathrm{M} \mathrm{pH6.8}$ Tris-HCl), heated for $5 \mathrm{~min}$ at $95^{\circ} \mathrm{C}$, separated via SDS-PAGE,

612 and transferred to nitrocellulose (Trans-blot turbo, Bio-Rad, Hercules, CA, USA).

613 Membranes were incubated with the appropriate primary (GLUT4 \#2213 and $\beta$-Actin

614 \#3700, Cell Signaling Technologies, Danvers, MA, USA) and fluorescent secondary

615 antibodies (IRDye 680 Goat anti-Mouse IgG, Li-Cor Bioscience, Lincoln, NE, USA).

616 Protein band fluorescence was quantified via Li-Cor Odessy Image studio Version 4.0

617 (Li-Cor Bioscience, Lincoln, NE, USA). Individual values are relative to the mean of all

618 sample values within the same membrane, and equal loading was confirmed and

619 normalized to the immuno-reactivity of $\beta$-Actin.

621 Data Analysis and Statistics

622 All data were analyzed and data plots were generated using Prism 6 (GraphPad

623 Software, Inc., La Jolla, CA, USA). Statistical comparisons were made using ANOVA

624 with post hoc analyses to compare groups. Tests with $p<0.05$ were deemed

625 statistically significant. Unless otherwise stated, all values plotted are presented as

626 mean $\pm S D$, with statistical results presented as ${ }^{*}=p<0.05,{ }^{* *}=p<0.01,{ }^{* *}=p<$

$627 \quad 0.001$ and $^{* * * *}=p<0.0001$. 


\section{AUTHOR CONTRIBUTIONS}

Experiments were designed by MHMdG, CMC, VK, JAM, and JST. Animal care and line maintenance was done by MHMdG. MHMdG, CMC, VK, JAM, and NIA performed the experiments and collected the data. Data analysis, interpretation and the generation of figures was done by MHMdG, CMC, VK, JAM and JST. The manuscript was written, reviewed and edited by MHMdG, CMC, VK, JAM and JST. JST directed and funded the research.

\section{ACKNOWLEDGMENTS}

Research was supported by the NIH (\#U01 MH 61915) and the Howard Hughes Medical Institute (JST). Additional funding was received by CMC from the NIDDK (F32DK104659). We would like to thank Delali Bassowou, Kelly Bruckmann, Kelly Foster, Kyung-Inn Kim, Izabela Kornblum, Sammie McMurray, Chris Olker, and Dawn Olson for technical assistance. We thank Sandy Siepka and Martha Vitaterna for production of the ENU mutagenized mice and design of the initial phenotypic screen. JST is a co-founder of, a Scientific Advisory Board member of, and a paid consultant for Reset Therapeutics, Inc., a biotechnology company aimed at discovering smallmolecule therapies that modulate circadian activity for a variety of disease indications. JST is an Investigator, and MHMdG. is a Research Specialist, in the Howard Hughes Medical Institute. 


\section{REFERENCES}

1. Jason LA, Evans M, Brown M, \& Porter N (2010) What is fatigue? Pathological and nonpathological fatigue. PM R 2(5):327-331.

2. Fukuda K, et al. (1994) The chronic fatigue syndrome: A comprehensive approach to its defintion and study. Ann Intern Med 121:953-959.

3. Wessely S (1998) The epidemiology of chronic fatigue syndrome. Epidemiol Psichiatr Soc 7(1):10-24.

4. Ranjith G (2005) Epidemiology of chronic fatigue syndrome. Occup Med (Lond) 55(1):13-19.

5. Hardy SE \& Studenski SA (2008) Fatigue predicts mortality in older adults. J Am Geriatr Soc 56(10):1910-1914.

6. Hardy SE \& Studenski SA (2008) Fatigue and function over three years among older adults. J Gerontol A Biol Sci Med Sci 63(12):1389-1392.

7. Moreh E, Jacobs JM, \& Stessman J (2010) Fatigue, function, and mortality in older adults. J Gerontol A Biol Sci Med Sci 65(8):887-895.

8. Maes M \& Twisk FNM (2010) Chronic fatigue syndrome: Harvey and Wessely's (bio)psychosocial model versus a bio(psychosocial) model based on inflammatory and oxidative and nitrosative stress pathways. BMC Medicine 8(35):1-13.

9. Harrington ME (2012) Neurobiological studies of fatigue. Prog Neurobiol 99(2):93-105.

10. Anisman H, Gibb J, \& Hayley S (2008) Influence of continuous infusion of interleukin-1beta on depression-related processes in mice: corticosterone, circulating cytokines, brain monoamines, and cytokine mRNA expression. Psychopharmacology (Berl) 199(2):231-244.

11. Kudo T, Loh DH, Truong D, Wu Y, \& Colwell CS (2011) Circadian dysfunction in a mouse model of Parkinson's disease. Exp Neurol 232(1):66-75.

12. Kudo T, et al. (2011) Dysfunctions in circadian behavior and physiology in mouse models of Huntington's disease. Exp Neurol 228(1):80-90.

13. Nakamura TJ, et al. (2011) Age-related decline in circadian output. J Neurosci 31(28):10201-10205. 
14. Ottenweller JE, et al. (1998) Mouse Running Activity Is Lowered by Brucella abortus Treatment: A Potential Model to Study Chronic Fatigue. Physiol Behav 63(5):795-801.

15. Ray MA, Trammell RA, Verhulst S, Ran S, \& Toth LA (2011) Development of a Mouse Model for Assessing Fatigue during Chemotherapy. Comp Medicine 61(2):119-130.

16. Bonsall DR, et al. (2015) Suppression of Locomotor Activity in Female C57BI/6J Mice Treated with Interleukin-1beta: Investigating a Method for the Study of Fatigue in Laboratory Animals. PLoS One 10(10):e0140678.

17. Lightfoot JT (2011) Current understanding of the genetic basis for physical activity. J Nutr 141(3):526-530.

18. Shimomura K, et al. (2001) Genome-wide epistatic interaction analysis reveals complex genetic determinants of circadian behavior in mice. Genome Res 11(6):959-980.

19. Siepka SM \& Takahashi JS (2005) Methods to record circadian rhythm wheel running activity in mice. Methods Enzymol 393:230-239.

20. Schur E, Afari N, Goldberg J, Buchwald D, \& Sullivan PF (2007) Twin analyses of fatigue. Twin Res Hum Genet 10(5):729-733.

21. Landmark-Hoyvik $\mathrm{H}$, et al. (2010) The genetics and epigenetics of fatigue. PM $R$ 2(5):456-465.

22. Sprangers MA, et al. (2014) Biological pathways, candidate genes, and molecular markers associated with quality-of-life domains: an update. Qual Life Res 23(7):1997-2013.

23. Wang T, Yin J, Miller AH, \& Xiao C (2017) A systematic review of the association between fatigue and genetic polymorphisms. Brain Behav Immun 62:230-244.

24. Narita M, et al. (2003) Association between serotonin transporter gene polymorphism and chronic fatigue syndrome. Biochem Biophys Res Commun 311(2):264-266.

25. Torpy DJ \& Ho JT (2007) Corticosteroid-binding globulin gene polymorphisms: clinical implications and links to idiopathic chronic fatigue disorders. Clin Endocrinol (Oxf) 67(2):161-167.

26. Collado-Hidalgo A, Bower JE, Ganz PA, Irwin MR, \& Cole SW (2008) Cytokine gene polymorphisms and fatigue in breast cancer survivors: early findings. Brain Behav Immun 22(8):1197-1200. 
27. Lin E \& Huang LC (2008) Identification of significant genes in genomics using Bayesian variable selection methods. Adv Appl Bioinform Chem 1:13-18.

28. Smith AK, et al. (2008) Genetic evaluation of the serotonergic system in chronic fatigue syndrome. Psychoneuroendocrinology 33(2):188-197.

29. Smith AK, Fang H, Whistler T, Unger ER, \& Rajeevan MS (2011) Convergent genomic studies identify association of GRIK2 and NPAS2 with chronic fatigue syndrome. Neuropsychobiology 64(4):183-194.

30. Johnston S, Staines D, Klein A, \& Marshall-Gradisnik S (2016) A targeted genome association study examining transient receptor potential ion channels, acetylcholine receptors, and adrenergic receptors in Chronic Fatigue Syndrome/Myalgic Encephalomyelitis. BMC Med Genet 17(1):79.

31. Schlauch KA, et al. (2016) Genome-wide association analysis identifies genetic variations in subjects with myalgic encephalomyelitis/chronic fatigue syndrome. Transl Psychiatry 6:e730.

32. Petersen $\mathrm{HH}$, et al. (2006) Hyporesponsiveness to glucocorticoids in mice genetically deficient for the corticosteroid binding globulin. Mol Cell Biol 26(19):7236-7245.

33. Golumbek PT, Keeling RM, \& Connolly AM (2007) RAG2 gene knockout in mice causes fatigue. Muscle Nerve 36(4):471-476.

34. Krzyszton CP, et al. (2008) Exacerbated fatigue and motor deficits in interleukin10-deficient mice after peripheral immune stimulation. Am J Physiol Regul Integr Comp Physiol 295(4):R1109-1114.

35. Justice MJ, Noveroske JK, Weber JS, Zheng B, \& Bradley A (1999) Mouse ENU Mutagenesis. Human Mol Genetics 8(10):1955-1963.

36. Soewarto D, et al. (2000) The large-scale Munich ENU-mouse-mutagenesis screen. Mamm Genome 11(7):507-510.

37. Keays DA \& Nolan PM (2003) N-ethyl-N-nitrosourea mouse mutants in the dissection of behavioural and psychiatric disorders. European Journal of Pharmacology 480(1-3):205-217.

38. Clark AT, et al. (2004) Implementing large-scale ENU mutagenesis screens in North America. Genetica 122(1):51-64.

39. Goldowitz D, et al. (2004) Large-scale mutagenesis of the mouse to understand the genetic bases of nervous system structure and function. Brain Res Mol Brain Res 132(2):105-115. 
40. Cordes SP (2005) N-ethyl-N-nitrosourea mutagenesis: boarding the mouse mutant express. Microbiol Mol Biol Rev 69(3):426-439.

41. Vitaterna MH, Pinto LH, \& Takahashi JS (2006) Large-scale mutagenesis and phenotypic screens for the nervous system and behavior in mice. Trends Neurosci 29(4):233-240.

42. Kumar V, et al. (2011) Second-generation high-throughput forward genetic screen in mice to isolate subtle behavioral mutants. Proc Natl Acad Sci U S A 108(3):15557-15564.

43. Moresco EM, Li X, \& Beutler B (2013) Going forward with genetics: recent technological advances and forward genetics in mice. Am J Pathol 182(5):14621473.

44. Takahashi JS, Shimomura K, \& Kumar V (2008) Searching for genes underlying behavior: lessons from circadian rhythms. Science 322(5903):909-912.

45. Siepka SM \& Takahashi JS (2005) Forward genetic screens to identify circadian rhythm mutants in mice. Methods Enzymol 393:219-229.

46. Katz EB, Stenbit AE, Hatton K, DePinho R, \& Charron MJ (1995) Cardiac and adipose tissue abnormalities but not diabetes in mice deficient in GLUT4. Nature 377(6545):151-155.

47. Gorselink M, et al. (2002) Increased muscle fatigability in GLUT-4-deficient mice. Am J Physiol Endocrinol Metab 282(2):E348-354.

48. Simoes MV, et al. (2004) Delayed response of insulin-stimulated fluorine-18 deoxyglucose uptake in glucose transporter-4-null mice hearts. J Am Coll Cardiol 43(9):1690-1697.

49. Fueger PT, et al. (2007) Glucose kinetics and exercise tolerance in mice lacking the GLUT4 glucose transporter. J Physiol 582(Pt 2):801-812.

50. Fam BC, et al. (2012) Normal muscle glucose uptake in mice deficient in muscle GLUT4. J Endocrinol 214(3):313-327.

51. Morris G, Berk M, Walder K, \& Maes M (2015) The Putative Role of Viruses, Bacteria, and Chronic Fungal Biotoxin Exposure in the Genesis of Intractable Fatigue Accompanied by Cognitive and Physical Disability. Mol Neurobiol 53(4):2550-2571.

52. Saligan LN, et al. (2015) The biology of cancer-related fatigue: a review of the literature. Support Care Cancer 23(8):2461-2478. 
53. Skoie IM, Ternowitz T, Jonsson G, Norheim K, \& Omdal R (2015) Fatigue in psoriasis: a phenomenon to be explored. Br J Dermatol 172(5):1196-1203.

54. Tsao TS, et al. (1997) Muscle-specific transgenic complementation of GLUT4deficient mice. Effects on glucose but not lipid metabolism. J Clin Invest 100(3):671-677.

55. Stenbit AE, et al. (2000) Preservation of glucose metabolism in hypertrophic GLUT4-null hearts. Am J Physiol Heart Circ Physiol 279(1):H313-318.

56. Jiang H, Li J, Katz EB, \& Charron MJ (2001) GLUT4 ablation in mice results in redistribution of IRAP to the plasma membrane. Biochem Biophys Res Commun 284(2):519-525.

57. Huang S \& Czech MP (2007) The GLUT4 glucose transporter. Cell Metab $5(4): 237-252$.

58. Sadler JB, Bryant NJ, Gould GW, \& Welburn CR (2013) Posttranslational modifications of GLUT4 affect its subcellular localization and translocation. Int J Mol Sci 14(5):9963-9978.

59. Govers R (2014) Cellular regulation of glucose uptake by glucose transporter GLUT4. Adv Clin Chem 66:173-240.

60. Alvim RO, Cheuhen MR, Machado SR, Sousa AG, \& Santos PC (2015) General aspects of muscle glucose uptake. An Acad Bras Cienc 87(1):351-368.

61. Gannon NP, Conn CA, \& Vaughan RA (2015) Dietary stimulators of GLUT4 expression and translocation in skeletal muscle: a mini-review. Mol Nutr Food Res 59(1):48-64.

62. Sylow L, Kleinert M, Richter EA, \& Jensen TE (2017) Exercise-stimulated glucose uptake - regulation and implications for glycaemic control. Nat Rev Endocrinol 13(3):133-148.

63. Rossetti L, et al. (1997) Peripheral but not hepatic insulin resistance in mice with one disrupted allele of the glucose transporter type 4 (GLUT4) gene. J Clin Invest 100(7):1831-1839.

64. Stenbit AE, et al. (1997) GLUT4 heterozygous knockout mice develop muscle insulin resistance and diabetes. Nat Med 3(10):1096-1101.

65. Tsao TS, et al. (1999) Prevention of insulin resistance and diabetes in mice heterozygous for GLUT4 ablation by transgenic complementation of GLUT4 in skeletal muscle. Diabetes 48(4):775-782. 
66. Li J, Houseknecht KL, Stenbit AE, Katz EB, \& Charron MJ (2000) Reduced glucose uptake precedes insulin signaling defects in adipocytes from heterozygous GLUT4 knockout mice. FASEB J 14(9):1117-1125.

67. Charron MJ, Brosius FC, Alper SL, \& Lodish HF (1989) A glucose transport protein expressed predominately in insulin-responsive tissues. Proc Natl Acad Sci USA 86:2535-2539.

68. Liu ML, et al. (1992) Expression and regulation of the human GLUT4/muscle-fat facilitative glucose transporter gene in transgenic mice. J Biol Chem 267(17):11673-11676.

69. Choeiri C, Staines W, \& Messier C (2002) Immunohistochemical localization and quantification of glucose transporters in the mouse brain. Neuroscience 111(1):19-34.

70. Ashrafi G, Wu Z, Farrell RJ, \& Ryan TA (2017) GLUT4 Mobilization Supports Energetic Demands of Active Synapses. Neuron 93(3):606-615 e603.

71. Lin HV, et al. (2011) Diabetes in mice with selective impairment of insulin action in Glut4-expressing tissues. Diabetes 60(3):700-709.

72. Reno CM, et al. (2017) Brain GLUT4 Knockout Mice Have Impaired Glucose Tolerance, Decreased Insulin Sensitivity, and Impaired Hypoglycemic Counterregulation. Diabetes 66(3):587-597.

73. Harno E, Cottrell EC, \& White A (2013) Metabolic pitfalls of CNS Cre-based technology. Cell Metab 18(1):21-28.

74. Ren H, et al. (2014) Glut4 expression defines an insulin-sensitive hypothalamic neuronal population. Mol Metab 3(4):452-459.

75. Ren H, Lu TY, McGraw TE, \& Accili D (2015) Anorexia and Impaired Glucose Metabolism in Mice With Hypothalamic Ablation of Glut4 Neurons. Diabetes 64:405-417.

76. Tsao TS, et al. (2001) Metabolic adaptations in skeletal muscle overexpressing GLUT4: effects on muscle and physical activity. FASEB J 15(6):958-969.

77. Dawes M, et al. (2014) Differential gene expression in high- and low-active inbred mice. BioMed research international 2014:361048.

78. Wang YX, et al. (2004) Regulation of muscle fiber type and running endurance by PPARdelta. PLoS Biol 2(10):e294.

79. Narkar VA, et al. (2008) AMPK and PPARdelta agonists are exercise mimetics. Cell 134(3):405-415. 
80. Fan W, et al. (2017) PPARdelta Promotes Running Endurance by Preserving Glucose. Cell Metab 25(5):1186-1193 e1184.

81. Jordan SD, et al. (2017) CRY1/2 Selectively Repress PPARdelta and Limit Exercise Capacity. Cell Metab 26(1):243-255 e246.

82. Fan W \& Evans RM (2017) Exercise Mimetics: Impact on Health and Performance. Cell Metab 25(2):242-247.

83. Fritschi C \& Quinn L (2010) Fatigue in patients with diabetes: a review. J Psychosom Res 69(1):33-41.

84. Lasselin J, et al. (2012) Fatigue and cognitive symptoms in patients with diabetes: relationship with disease phenotype and insulin treatment. Psychoneuroendocrinology 37(9):1468-1478.

85. Moulton CD, Pickup JC, \& Ismail K (2015) The link between depression and diabetes: the search for shared mechanisms. Lancet Diabetes Endocrinol $3(6): 461-471$.

86. Germain A, Ruppert D, Levine SM, \& Hanson MR (2017) Metabolic profiling of a myalgic encephalomyelitis/chronic fatigue syndrome discovery cohort reveals disturbances in fatty acid and lipid metabolism. Mol Biosyst 13(2):371-379.

87. Klimas NG, Broderick G, \& Fletcher MA (2012) Biomarkers for chronic fatigue. Brain Behav Immun 26(8):1202-1210.

88. Maxmen A (2017) Biological underpinnings of chronic fatigue syndrome begin to emerge. Nature 543(7647):602.

89. Pack Al, et al. (2007) Novel method for high-throughput phenotyping of sleep in mice. Physiol Genomics 28(2):232-238.

90. Kumar V, et al. (2013) C57BL/6N mutation in cytoplasmic FMRP interacting protein 2 regulates cocaine response. Science 342(6165):1508-1512.

91. Broman KW, Wu H, Sen S, \& Churchill GA (2003) R/qtl: QTL mapping in experimental crosses. Bioinformatics 19(7):889-890.

92. Li H \& Durbin R (2009) Fast and accurate short read alignment with BurrowsWheeler transform. Bioinformatics 25(14):1754-1760.

93. McKenna A, et al. (2010) The Genome Analysis Toolkit: a MapReduce framework for analyzing next-generation DNA sequencing data. Genome Res 20(9):1297-1303. 
94. Cingolani $\mathrm{P}$, et al. (2012) A program for annotating and predicting the effects of single nucleotide polymorphisms, SnpEff: SNPs in the genome of Drosophila melanogaster strain w1118; iso-2; iso-3. Fly (Austin) 6(2):80-92.

95. Fujikawa T, et al. (2016) SF-1 expression in the hypothalamus is required for beneficial metabolic effects of exercise. Elife 5. 


\section{FIGURE LEGENDS}

Fig. 1. Mice from two lines show abnormal, low amplitude voluntary wheel-running activity patterns. (A) Representative actogram of a B/Int-x (N6F2) mouse showing a normal circadian activity pattern, double plotted for ease of visualization, with each horizontal line representing $48 \mathrm{~h}$ and successive days (1-96) plotted along the $\mathrm{Y}$-axis. Wheel revolutions are depicted as black marks on the horizontal lines. Mice were initially recorded under a $12 \mathrm{~h}$ light:12h dark (LD) cycle shown in the bar across the top of the graph (black = dark phase). After 3 weeks, mice were recorded in constant darkness (DD) for 3 weeks, in 12:12 LD for 3 weeks, in constant light (LL) for 2 weeks and a final 2 weeks in DD (indicated on right $Y$-axis). Wheel running activity shows a normal nocturnal pattern during recording under LD with high levels of activity concentrated in the dark phase and low activity during the light phase. In DD, the rhythm free-runs with a period of $\sim 23.7 \mathrm{~h}$, re-entrains normally upon reintroduction of the LD cycle, and free-runs with a lengthened period under LL. Colored vertical bars on the left side of the actogram indicate days used for quantification of wheel-running behavior. Representative actogram of a B/Int-x (N6F2) (B) and two I-x (N2F10) (C\&D) mice showing an abnormal circadian activity pattern with plotting conventions and recording conditions as described for (A). (E) Representative actograms of two T-x (N3) mice recorded for 3 weeks in LD followed by 3 weeks in DD. Plotting conventions are as described for $(A)$. (F) Average waveforms for activity recorded in LD for mice in the $\mathrm{B} / \mathrm{IntX}(\mathrm{N} 6 \mathrm{~F} 2 ; \mathrm{n}=40)$, I-x (N2F10; $\mathrm{n}=26)$, and T-x (N3; $\mathrm{n}=27$ ) groups. Time (24h) is plotted along the $X$-axis and average number of wheel revolutions $( \pm S E M)$ is plotted along the Y-axis. The LD cycle is shown in the bar across the bottom of the graph with lights off from 12 to 24 indicated in black. Average number of wheel revolutions per min over $24 \mathrm{~h}(\mathrm{G}), \%$ of daily activity occurring in the light phase $(\mathrm{H})$, and number of discreet bouts of activity occurring each day (I) during recording under LD for mice in the B/IntX (N6F2; $n=40), I-x(N 2 F 10 ; n=26)$, and T-x (N3; n=27) groups are plotted. Average number of wheel revolutions per min over $24 \mathrm{~h}(\mathrm{~J})$, amplitude $(\mathrm{K})$ and period $(\mathrm{L})$ of the wheel-running rhythm recorded in DD for these same mice is also shown. Means $\pm S D$ with individual data points are plotted in $\mathrm{G}-\mathrm{L}$ with significant differences among groups indicated $\left({ }^{*}=p<0.05,{ }^{* *}=p<0.01,{ }^{* * *}=p<0.001,{ }^{* * *}=p<0.0001\right)$.

Fig. 2. Nocturnal wheel-running activity is interrupted by frequent rest bouts in affected mice. Ethogram depicting behaviors, states or locations observed in images captured once every min for $15 \mathrm{~h}$ beginning at lights off for a wildtype C57BL/6J (A) and an I-x (N2F14) (B) mouse, with time plotted along the X-axis. Each photograph was scored on a 9 item scale using the following designations: 1) drinking, 2) eating, 3) near hopper, 4) running on wheel, 5) near wheel awake, 6) end awake, 7) end resting, 8) near wheel asleep, and 9) end asleep, where 'end' refers to the end of the cage furthest away from the wheel. Color code for each score is shown in the legend below (B). Pie charts 
showing the amount of time spent in each of the 9 behavioral states for the first 6 h of recording for the individual plotted in $A(C)$, and averaged for all 3 wildtype C57BL/6J mice (D), the individual plotted in $B(E)$, and averaged for all $10 \mathrm{l}-\mathrm{x}(\mathrm{N} 2 \mathrm{~F} 14 / 15)$ mice $(F)$ are shown. Note: 5 individual I-x (N2F14/15) mice were recorded twice each with one week between recording days. Pictures in $(\mathrm{G})$ show a representative mouse scored as "near wheel awake" (right) and "near wheel asleep" (left). See also data for individual mice plotted in Fig. S2 and S3.

Fig. 3. Video recording shows bouts of sleep during active phase in affected mice. Total number of wheel revolutions recorded for $2 \mathrm{~h}$ to $3.5 \mathrm{~h}$ after lights off for a T-x (N3F2) mouse and 3 individual I-x (N2F21) mice (A), and a different T-x (N3F2) and 3 individual T-x (N11F3) mice (B) are plotted, with periods of prolonged immobility consistent with sleep indicated in colored bars along the X-axis below. Periods $>40$ s of total immobility with low posture were scored as sleep.

Fig. 4. Abnormal phenotypes map to locus on chromosome 11. Representative actograms of an unaffected $(A)$ and two affected (B \& C) M-x (F2) mice, which were recorded for 3 weeks in LD followed by 3 weeks in DD. Plotting conventions are as those described for Fig. 1A. Average number of wheel revolutions per min recorded in LD (D) or DD (E) and amplitude of the free-running rhythm in DD (F) for mice in the WTB6B10 (F1; n=63), WTB6B10 (F2; n=112), M-x (F1; n=92) and M-x (F2; n=207) groups are shown. Genome wide linkage plots for average number of wheel revolutions per min recorded in LD $(\mathrm{G})$ or DD $(\mathrm{H})$ and amplitude of the free-running rhythm in DD $(\mathrm{I})$ all show linkage to a locus on chromosome 11, with chromosomal location plotted along the X-axis. Average number of wheel revolutions per min recorded in LD (J), or DD (K) and amplitude of the free-running rhythm in DD (L) for mice with B6/B6 ( $\mathrm{n=26}$ ), B6/B10 $(n=33)$, or B10/B10 $(n=14)$ genotypes at a SNP marker ( $r$ 13481117) located on chromosome 11 at position 79252230 are plotted. Means \pm SD and individual data points are plotted and significant differences among groups are indicated $\left(^{*}=p<0.05\right.$, $\left.{ }^{* *}=p<0.01,{ }^{* * *}=p<0.001,{ }^{* * * *}=p<0.0001\right)$. See Fig. S4 for related data.

Fig. 5. Affected mice harbor a single point mutation in Slc2a4 encoding the GLUT4 insulin-responsive glucose transporter. Results of whole-exome sequencing of spleen genomic DNA reveal a single amino acid change (A to T) at position 69944065 within the Slc2a4 gene on chromosome $11(\mathrm{~A})$, indicated with a red arrow on the coverage plots. All reads at this locus for the I-x (N2F20) and the T-x (N11F3) samples ( $\mathrm{n}=16$ per group, pooled) showed this single nucleotide alteration (indicated by red line), and 19 representative reads from each sample are shown at higher magnification at the bottom of the plot. A cartoon of the GLUT4 insulin-responsive glucose transporter with its 12 trans-membrane domains is shown in (B) with the A to T SNP location and resulting 
premature stop (Y440*) in exon 10 indicated with a red line. The mutation falls within the last extracellular loop and likely leads to a truncation of the cytoplasmic C-Terminus domain (indicated in grey). Protein abundance of GLUT4 in the gastrocnemius muscle of representative wildtype $(+/+)$ and homozygous mutant (twgy/twgy) mice is shown in $\mathrm{C}$ with means \pm SD and individual data points of $+/+$ or + /twgy $(n=16)$ and twgy/twgy $(n=14)$ plotted below. ${ }^{* * * *}$ indicates significant difference between genotypes $(p<$ 0.0001). See Fig. S5 for related data.

Fig. 6. The twiggy mutation of Slc2a4 results in impaired glucose tolerance, increased heart weight, low body weight, and decreased exercise tolerance. (A) Glucose tolerance response for C57BL/6J $(n=13)$, wildtype $(+/+; n=6)$, heterozygous (+/twgy; $n=27)$ and homozygous (twgy/twgy; $\mathrm{n}=56$ ) mice. Blood glucose (mg/dL $\pm \mathrm{SEM}$ ) is plotted on the $\mathrm{Y}$ axis with time since I.P. injection of glucose plotted along the $\mathrm{X}$-axis. Significant differences between twgy/twgy and C57BL/6J at 15, 30 and $45 \mathrm{~min}$ is indicated ${ }^{* *}=\mathrm{p}<$ $\left.0.01,{ }^{* * *}=p<0.0001\right) .+/+$ and $+/$ twgy did not differ from C57BL/6J at any time point. (B) Blood glucose $(\mathrm{mg} / \mathrm{dL} \pm \mathrm{SD}$ ) following a $4 \mathrm{~h}$ fast in the middle of the light phase for male (blue, left) and female (red, right) C57BL/6J (B6; $n=12 M \& n=5 F),+/+(n=4 M$ \& $n=7 F),+$ twgy $(n=15 M \& n=16 F)$ and twgy/twgy $(n=36 M \& n=38 F)$ is plotted. (C) Body weights of the same mice shown in $B$ taken after a $4 \mathrm{~h}$ fast. Heart weights (as a percentage of total body weight) of male (blue, left) and female (red, right) C57BL/6J $(B 6 ; n=10 M \& n=5 F),+/+(n=5 M \& n=8 F),+$ twgy $(n=18 M \& n=13 F)$ and twgy/twgy $(n=36 M \& n=39 F)$ mice are shown in (D). A treadmill exercise test run on $+/+(n=3 M$ \& $n=3 F)$, +/twgy ( $n=16 M$ \& $n=4 F)$ and twgy/twgy ( $n=6 M$ \& $n=9 F)$ mice showed impaired responses in homozygous twgy/twgy mice in time (in min; E), distance (in $\mathrm{m}$; F), blood glucose $(\mathrm{G})$ and lactate $(\mathrm{H})$ at exhaustion. Means $\pm \mathrm{SD}$ and individual data points are plotted with significant differences indicated $\left({ }^{*}=p<0.05,{ }^{* *}=p<0.01,{ }^{* \star *}=p<0.001\right.$, $\star * * *=p<0.0001)$.

Fig. 7. Slc2a $4^{\text {twgy/twgy }}$ mice show abnormal, low-amplitude circadian patterns of voluntary wheel-running behavior interrupted with frequent rest bouts. (A) Representative actograms of B/Int-x (N12F2) mice of all three genotypes (+/+ - top, +/twgy - middle, twgy/twgy - bottom) recorded for 3 weeks under a full 12:12 LD cycle (shown in bar across the top). Plotting conventions are like those for Fig 1A. Colored vertical bars on the left side of the actograms indicate days used for quantification of wheel-running behavior. Average number of wheel revolutions per min over $24 \mathrm{~h}(\mathrm{~B})$, and \% of daily activity occurring in the light phase $(C)$ for mice in the $+/+(n=4 M \& n=5 F),+/$ twgy $(n=19 M \& n=16 F)$, and twgy/twgy $(n=41 M \& n=45 F)$ groups are plotted. Means $\pm S D$ and individual data points are plotted with significant differences indicated $\left({ }^{\star *}=p<0.01\right.$, $\left.{ }^{\star * *}=p<0.001,{ }^{* \star * *}=p<0.0001\right)$. 


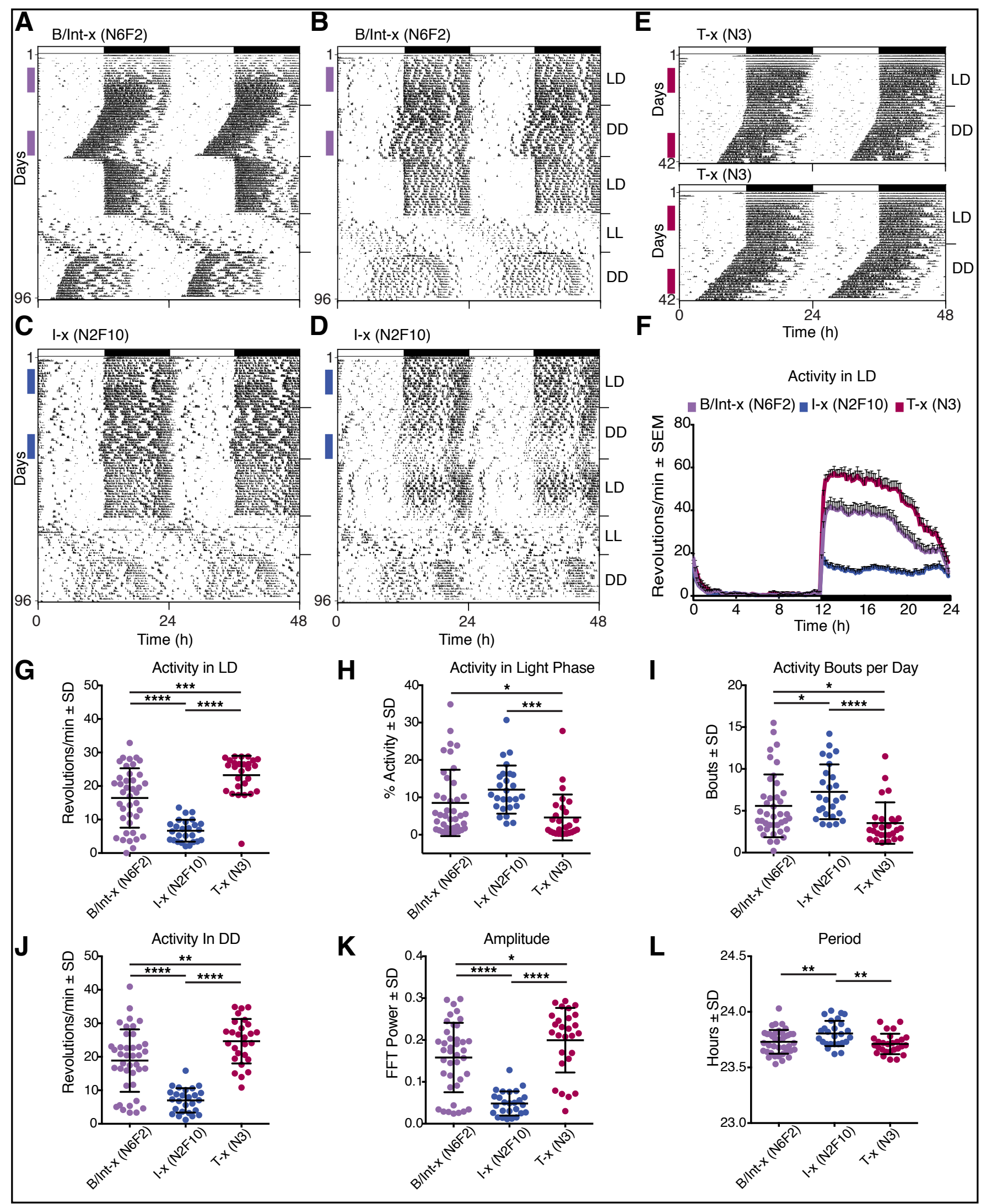

Figure 1 


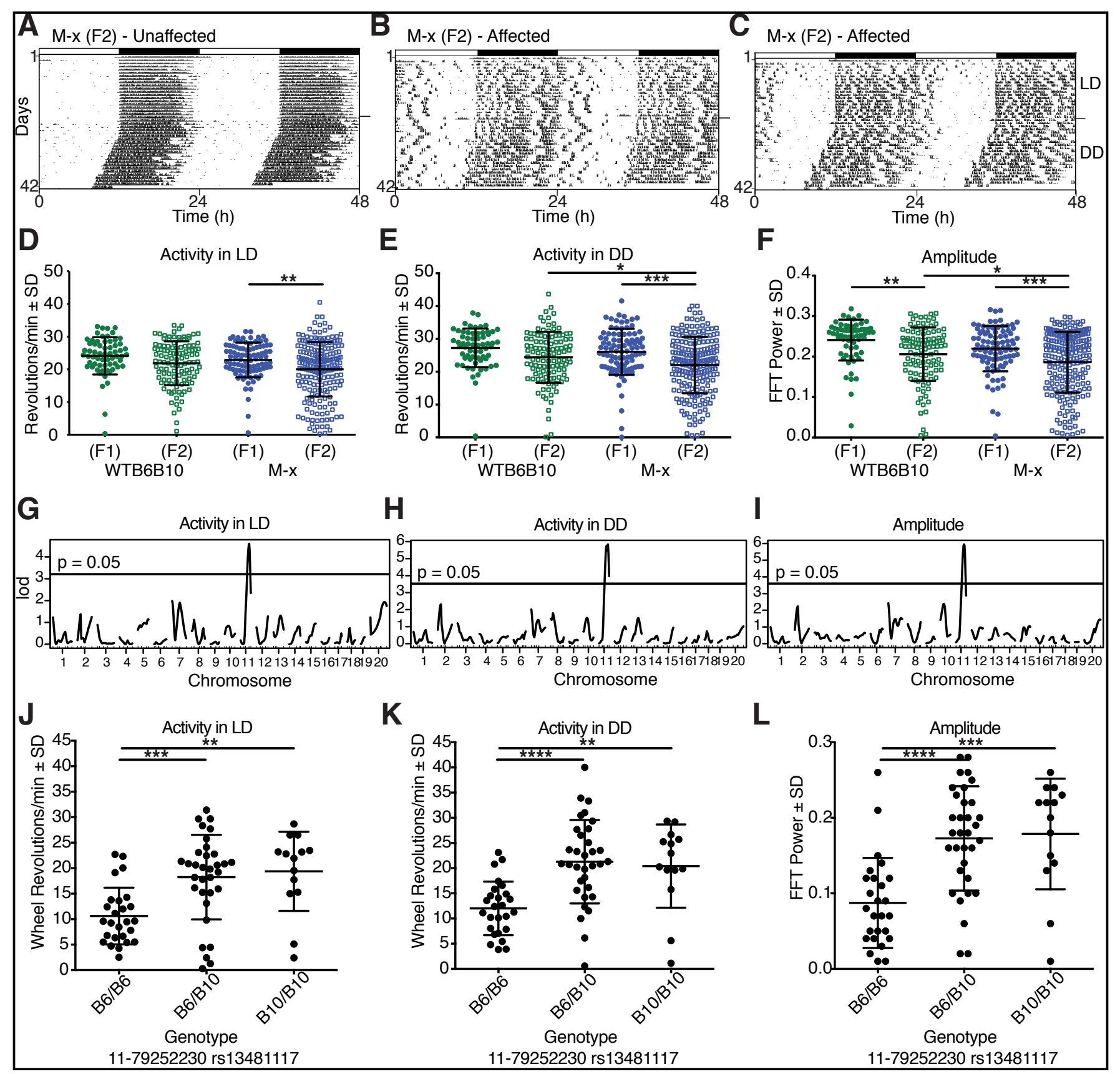

Figure 4 


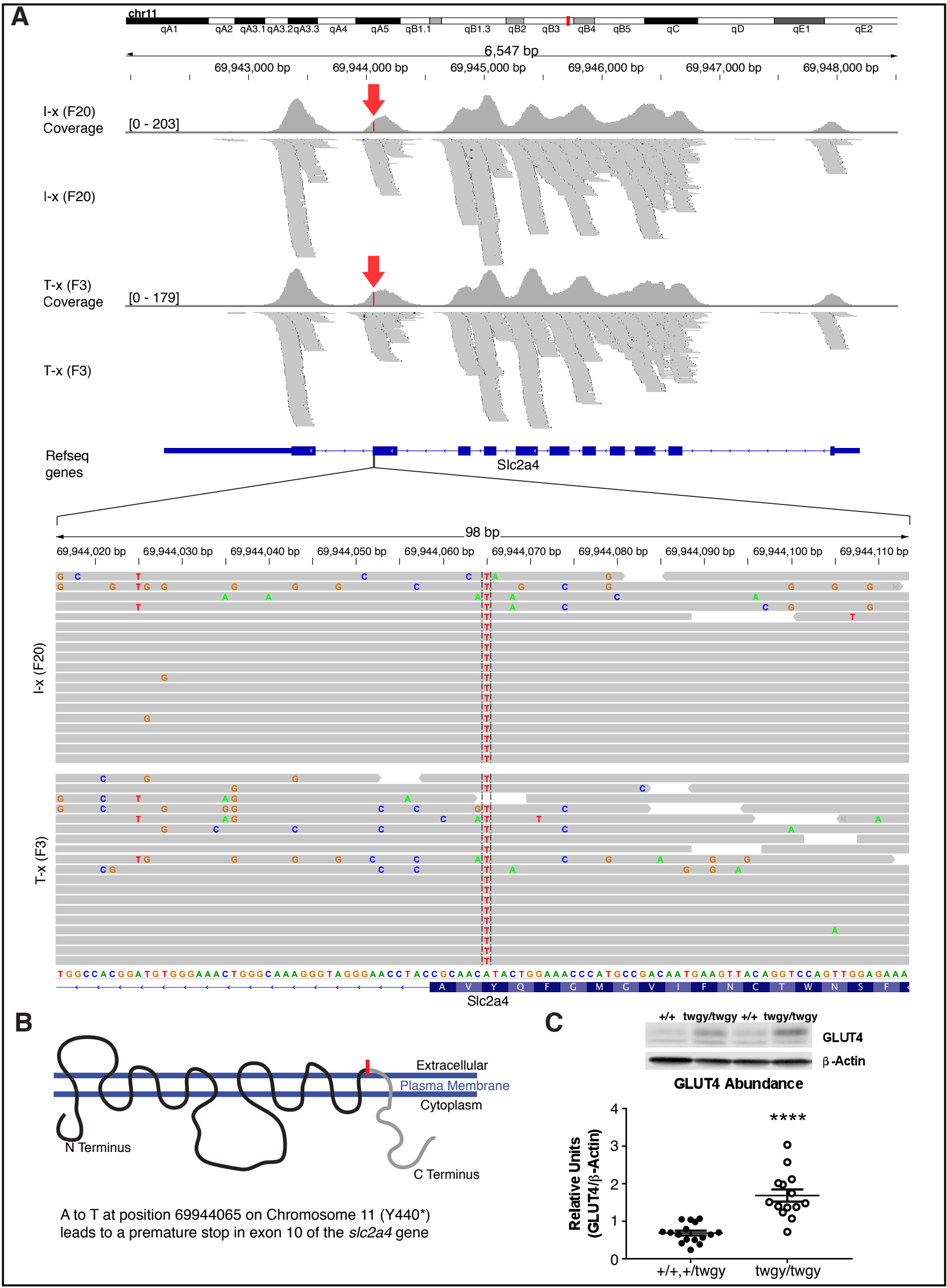

Figure 5 


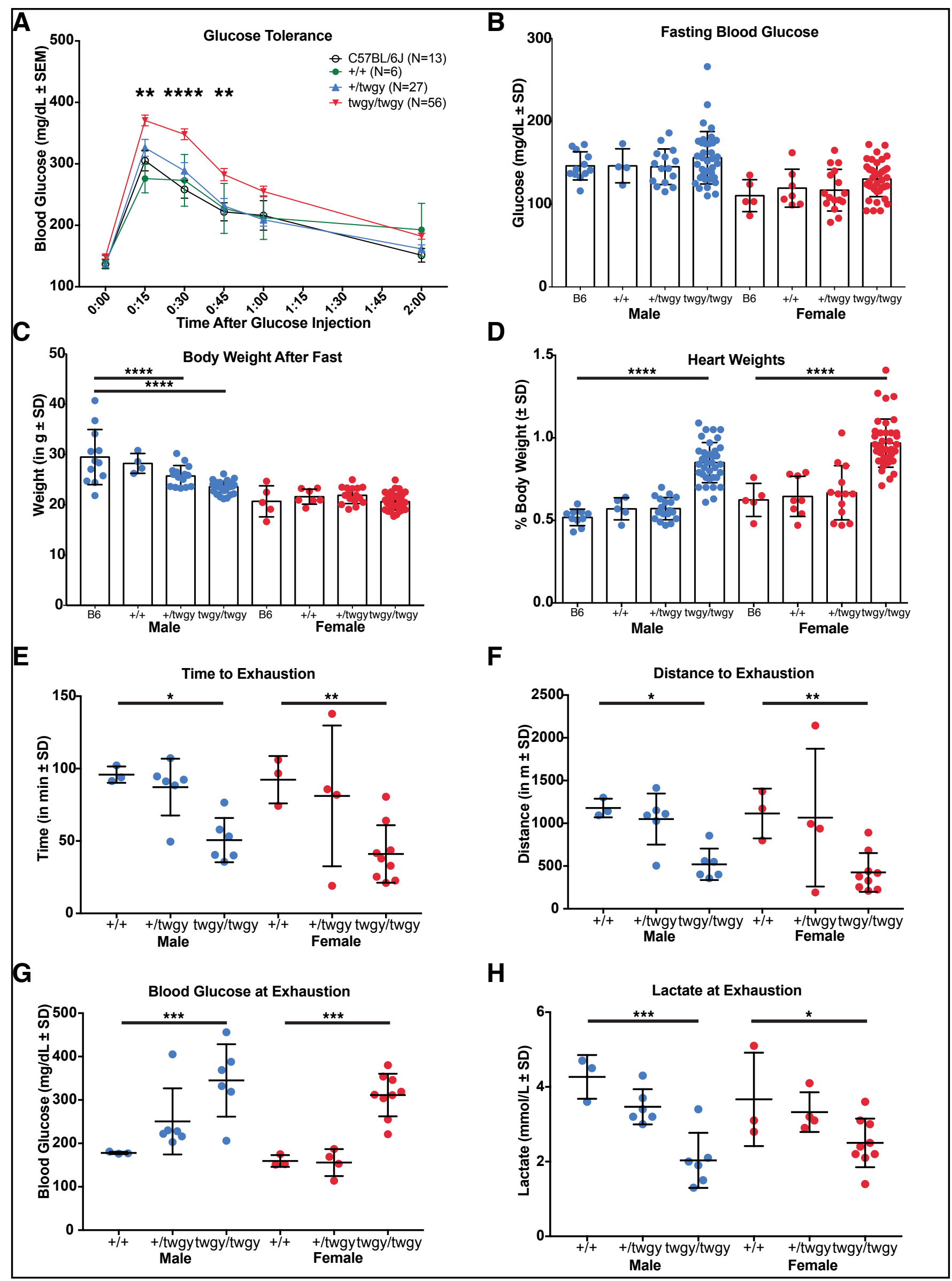




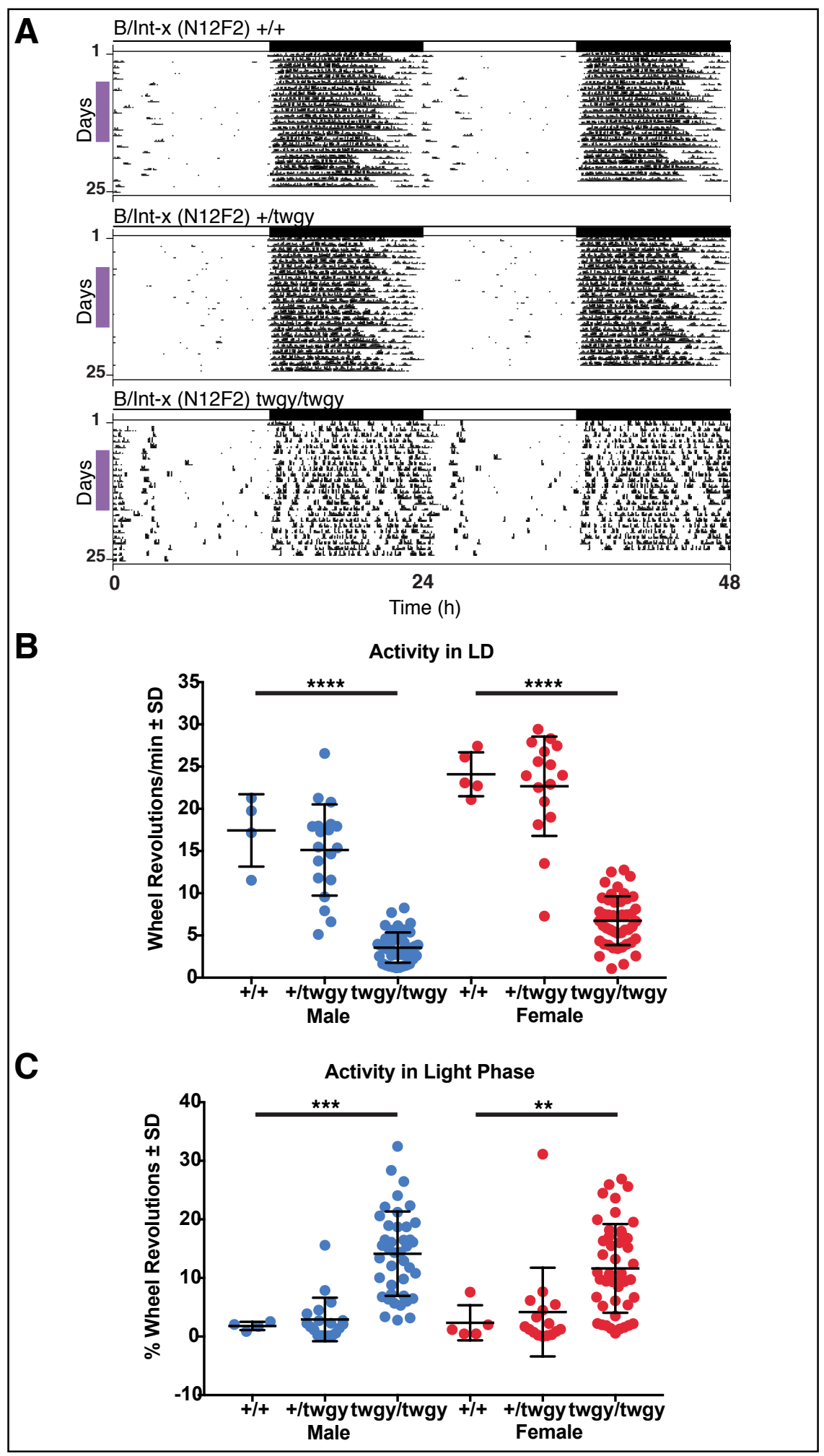

Figure 7 